\title{
Editorial
}

\section{Luteolin: A potential flavonoid for cancerous diseases}

\section{Dr. Santram Lodhi}

\author{
Department of Pharmacognosy, \\ Smt. Sharadchandrika Suresh Patil College of Pharmacy, Chopda, Jalgaon 425107, M. S. India.
}

Cancer is a major worldwide health problem in both developed and developing countries. Several natural anti-cancer agents including taxol, vinblastine, vincristine, topotecan, irinotecan, etoposide etc. are clinically used. Some other major anti-cancer agents are including flavopiridol, roscovitine, betulinic acid and silvestrol. Among the various phytochemicals, particular flavonoids, are one of the most effective chemical classes which possess a wide range of health-promoting activities and pharmacological effects (Batra and Sharma, 2013).<smiles></smiles>

Figure 1. Luteolin (3,4,5,7-tetrahydroxy flavone)

Luteolin (3,4,5,7-tetrahydroxy flavone) is an important flavone, which is naturally found in several plant species. Chemically, it has a C6-C3-C6 structure that contains two benzene rings and one oxygen-containing ring with a $\mathrm{C} 2-\mathrm{C} 3$ carbon double bond (Bravo, 1998). Structure-activity studies have shown that the presence of hydroxyl moieties at carbons $5,7,3$ and 4 positions of the luteolin structure and the presence of the 2-3 double bond are responsible for its multiple pharmacological effects (Lin et al., 2008). Luteolin, which is naturally found as a glycosylated form, is present in different fruits and vegetables, including broccoli, pepper, thyme, and celery (Nabavi et al., 2015). Luteolin possesses antioxidant, anticancer, anti-inflammatory, and neuroprotective effects.

*Address for Corresponding Author:

Dr. Santram Lodhi

Department of Pharmacognosy,

Smt. Sharadchandrika Suresh Patil College of Pharmacy, Chopda,

Jalgaon 425107, M. S. India.

Email:srlodhi78@gmail.com
The bioavailability of luteolin needs to be sufficiently high and its metabolism sufficiently low; otherwise, many activities of luteolin observed in vitro will not be relevant in vivo. Since luteolin is a common dietary constituent and since the oral route is the preferred route of administration for most drugs, it is important to know the bioavailability and metabolism of this flavonoid after oral ingestion (López-Lázaro, 2009).

Luteolin and its glycosides are widely distributed in the plant kingdom and have been found in many edible plants. Numerous preclinical studies have shown that luteolin possesses a wide range of biological activities and several possible mechanisms of action have been elucidated. Although the oral bioavailability of luteolin and its glycosides is not too high, animal experiments have shown that luteolin exerts its biological properties in vivo. Accumulating evidence suggests that luteolin could be developed as a potential anti-inflammatory agent as well as cancer chemopreventive agent and be useful in cancer therapy to sensitize tumor cells to the cytotoxic effects of some chemotherapeutic drugs. It is suggested that longterm animal toxicity studies should be conducted before people are able to take high doses of luteolin safely over a long period of time.

Luteolin suppresses JNK in macrophages while it activates this kinase in cancer cells. Also, luteolin suppresses NF- $\kappa \mathrm{B}$ through inhibiting IKK activation during inflammation in epithelial cells and macrophages. However, in cancer cells suppression of NF- $\mathrm{KB}$ by luteolin is apparently a nuclear event. It remains to be determined whether the distinct mechanisms are due to differences in cell contexts. Because luteolin inhibits NF- $\kappa \mathrm{B}$ in lung cancer cells and is associated with its pro-oxidant effect, it will be interesting to determine if the distinct mechanisms in NF- $\kappa B$ suppression are dependent on the redox status of the cell or the redox-regulating function of luteolin (Lin et al., 2008). Luteolin significantly reduces VRK1-mediated

DOI: https://doi.org/10.31024/apj.2018.3.3.1

2456-1436/Copyright (C) 2018, N.S. Memorial Scientific Research and Education Society. This is an open access article under the CC BY-NC-ND license (http://creativecommons.org/licenses/by-nc-nd/4.0/). 
phosphorylation of the cell cycle-related substrates BAF and histone $\mathrm{H} 3$, and directly interacts with the catalytic domain of VRK1. In addition, luteolin regulates cell cycle progression by modulating VRK1 activity, leading to the suppression of cancer cell proliferation and the induction of apoptosis (Kim et al., 2014). Luteolin also acts as an antiproliferative by suppressing receptor tyrosine-kinase activity and apoptosis, in breast cancer. Many of these antimetastatic characteristics accredited to luteolin are likely functionally related (Cook, 2018). These findings suggest that luteolin can be used as a potential molecule for treatment of different types of cancer diseases.

\section{References}

Batra P, Sharma AK. 2013. Anti-cancer potential of flavonoids: recent trends and future perspectives. 3 Biotech 3(6):439459.

Bravo L. 1998. Polyphenols: chemistry, dietary sources, metabolism, andnutritional significance. Nutrition Reviews 56(11):317-333.

Cook MT. 2008. Mechanism of metastasis suppression by luteolin in breast cancer. Breast Cancer: Targets and Therapy 10:89-100.

Kim YS, Kim SH, Shin J, Harikishore A, Lim JK, Jung Y, Lyu HN, Baek NI, Choi KY, Yoon HS, Kim KT. 2014. Luteolin suppresses cancer cell proliferation by targeting vacciniarelated kinase 1.PLoS One 9(10):e109655.

Lin Y, Shi R, Wang X, Shen H-M. 2008. Luteolin, a flavonoid with potentials for cancer prevention and therapy. Current cancer drug targets 8(7):634-646.

Lin Y, Shi R, Wang X, Shen HM. 2008. Luteolin, a flavonoid with potentials forcancer prevention and therapy. Current Cancer Drug Targets 8(7):634-646.

López-Lázaro M, 2009. Distribution and Biological Activities of the Flavonoid Luteolin. Mini-Reviews in Medicinal Chemistry 9:31-59.

Nabavi SF, Braidy N, Gortzi O, Sobarzo-Sanchez E, Daglia M, Skalicka-Woźniak K, Nabavi SM. 2015. Luteolin as an antiinflammatory and neuroprotective agent: A brief review. Brain Research Bulletin 119:1-11.

Odontuya G, Hoult JR, Houghton PJ. 2005. Structure-activity relationship for antiinflammatory effect of luteolin and its derived glycosides. Phototherapy Research 19:782-6. 